\title{
以苯丙氨酸为例说明中心离子在手性识别中的 作用
}

\author{
于擎，曹洁*，陆刚，汪志祥*
}

中国科学院研究生院, 北京 100049

* 通讯作者, E-mail: jcao@gucas.ac.cn, zxwang@gucas.ac.cn

收稿日期：2008-11-25；接受日期：2009-01-09

\begin{abstract}
摘要采用手性冠醚 (+)-2,3,11,12-四羧基-18-冠-6(简写为 18-C-6-TCA)作为手性 参照物, 分别以 $\mathrm{Cu}^{2+}$ 和 $\mathrm{H}^{+}$为中心离子, 对苯丙氨酸 $(\mathrm{Phe})$ 对映体进行手性识别研究. 通过离子键合二聚体 $\left[\mathrm{Cu}^{2+}(18-\mathrm{C}-6-\mathrm{TCA})(\mathrm{Phe})-\mathrm{H}^{+}\right]^{+}$和 (18-C-6-TCA)(Phe) $\mathrm{H}^{+}$的碰 撞诱导解离(CID)反应, 总结了上述非对映异构体离子的裂解特征并以其初级产物 离子与母离子的相对丰度为依据对Phe对映体进行手性识别. 通过实验发现, 作为过 渡金属代表的 $\mathrm{Cu}^{2+}$ 离子对多齿型配体具有较强的配合能力, $\mathrm{Cu}^{2+}$ 与 $\mathrm{Phe}$ 对映体形成 的非对映异构离子 $\left[\mathrm{Cu}^{2+}(18-\mathrm{C}-6-\mathrm{TCA})(\mathrm{Phe})-\mathrm{H}^{+}\right]^{+}$离子的稳定性差异较大, 手性识别 能力较强。同时, $\left[\mathrm{Cu}^{2+}(18-\mathrm{C}-6-\mathrm{TCA})(\mathrm{Phe})-\mathrm{H}^{+}\right]^{+}$的 $\mathrm{CID}$ 反应只产生 $\left[\mathrm{Cu}^{2+}(18-\right.$ C-6-TCA)- $\left.\mathrm{H}^{+}\right]^{+}$产物离子, 而 (18-C-6-TCA)(Phe) $\mathrm{H}^{+}$的 CID 反应除了产生质子化的 18-C-6-TCA以外, 还有冠梄离子的次级碎片产物. 这说明 18-C-6-TCA、 $\mathrm{Phe}^{-} \mathrm{Cu}^{2+}$ 的键合情况以及亲和势都与 $\mathrm{H}^{+}$的情形完全不同. 本文将结合量化计算对这一点进 行详细讨论.
\end{abstract}

\section{关键词}

手性冠梄

手性识别

非对映异构体

碰撞诱导解离反应

量化计算
手性是自然界一种普遍而有趣的现象，广泛存 在于物理、化学、材料、医药等各个学科领域中. 随 着对手性问题认识的逐步深入，人们已经认识到并 开始重视对映体之间的生理作用和代谢过程的差别. 由于生物体的手性环境, 当手性药物进入生物体后, 它的两种对映异构体通常会表现出不同甚至截然相 反的生物活性, 一种对映体是有效的, 而另一个对映 体则可能是无效或有毒的, 如沙利度胺 (Thalidomide $)^{[1]}$. 手性分离分析已成为当今分析科学 中的重要研究课题. 目前常用的手性分析方法主要 有旋光测定法 ${ }^{[2]}$ 、圆二色谱法 ${ }^{[3]}$ 、高效液相色谱法 ${ }^{[4]}$ 、 气相色谱法 ${ }^{[5]}$ 、毛细管电泳法 ${ }^{[6]}$ 等. 质谱法具有快速、 灵敏、专一并能直接给出化学计量比等特点 ${ }^{[7]}$, 同时
由于电喷雾电离(ESI)温和的电离过程使得以弱非共 价键结合的复合物可以完整地被检测出来，使得质 谱法得以广泛地应用于手性分析领域的研究. 手性 冠醚除具有冠醚的一般性质外, 主要用于立体化学 反应的研究，是常见的手性主体化合物之一，早期多 用于基于主客体交换法的伯胺类物质的手性识 别 $\frac{[8 \sim 10]}{}$.

手性质谱起源于 1977 年, 当时Fales ${ }^{[11]}$ 首次报 道了在质谱中发现了光学活性的二烷基酒石酸的手 性识别效应. 到目前为止已经有数十篇有关手性质 谱研究的文章发表 ${ }^{[1225]}$. 值得一提的是, 我们国内的 质谱学家 $[26$ 30] 也对这一领域开展了较深入和系统的 研究, 尤其是郭寅龙研究组 ${ }^{[31]}$ 通过对二-O-苯甲酰 
酒石酸二丁酯的手性识别研究，阐述了不同离子 $\left(\mathrm{H}^{+}\right.$、 $\mathrm{Zn}^{2+}$ 和 $\mathrm{Cu}^{2+}$ )对于该体系手性识别的重要作用.

到目前为止，质谱的手性识别方法主要有三种: (1) 主客体交换反应. Lebrilla 研究组 ${ }^{[20 ~ 25]}$ 采用了该 方法开展了一系列的工作. 他们选用全甲基 $\beta$-环糊精 和手性药物对映体形成的复合物离子与正丙胺分子 进行的主客体交换反应，一对对映体的反应速率常 数不同从而达到手性识别; (2) 动力学方法. 该方法是 目前质谱手性分析中使用最多的方法 ${ }^{[18]}$. Cooks 研究 组 ${ }^{[12 ~ 14,16,17,32]}$ 是通过观察过渡金属 $(\mathrm{M})$ 离子与手性分 子(A)以及另一种手性参比配体(Ref)形成的气相三聚 体复合物离子 $\left[\mathrm{M}(\mathrm{Ref})_{2}(\mathrm{~A})-\mathrm{H}\right]^{+}$在CID条件下的一对 竞争解离反应, 测量其二聚体产物离子 $[\mathrm{M}(\mathrm{Ref})$ $(\mathrm{A})-\mathrm{H}]^{+}$和 $\left[\mathrm{M}(\mathrm{Ref})_{2}-\mathrm{H}\right]^{+}$的相对丰度来表征两个竞争 反应的反应速率常数之比，一对对映该比值不同从 而实现对手性分子 $\mathrm{A}$ 的识别; (3) 手性离子/分子结合 法 ${ }^{[19,33 ~ 35]}$. 该方法主要是基于对映体分子与手性配 体形成的非对映异构体复合物的稳定性不同，在一 级质谱图上表现出该复合物离子相对丰度不同.

本文将基于动力学方法, 通过离子键合二聚体 $\left(\mathrm{M}=\mathrm{H}^{+}\right.$或 $\left.\mathrm{Cu}^{2+}\right)$ 的CID反应, 测量初级产物离子与 母离子的相对丰度 ${ }^{[36 ~ 38]}$ 来表示母离子的解离效率(即 母离子的稳定性)由Phe对映体: 18-C-6-TCA和不同中 心离子组成的二聚体离子, 其解离反应特征和解离 效率不同从而实现手性识别, 研究的主要目的是说 明不同中心离子的手性识别效果.

\section{1 实验部分}

\section{1 仪器与试剂}

美国 Waters 公司的 Q-TOF 质谱仪, 配有电喷雾 电离源. (+)-2,3,11,12-四羧基-18-冠-6 购自 Sigma 公司; $D 、 L$-苯丙氨酸购自百灵威公司; 甲醇(色谱纯)购自 Fisher 公司; 所用水为去离子蒸馏水.

\section{2 溶液的配制}

将适量的 (+)-2,3,11,12- 四羧基 - 18 - 冠 -6 用 $50 / 50(V / V)$ 的水/甲醇配制成 $0.1 \mathrm{~mol} / \mathrm{L}$ 的储备液; 将 $D 、 L$-苯丙氨酸分别用 $50 / 50(V / V)$ 的水/甲醇配制成 0.1 $\mathrm{mol} / \mathrm{L}$ 的储备液. 取适量的 $(+)-2,3,11,12$-四羒基-18冠-6 储备液分别与适量的 $D 、 L$-苯丙氨酸混合, 再加
入 $\mathrm{CuSO}_{4}$ 溶液, 使得最终待测液浓度为 $(+)-2,3,11$, 12-四羧基-18-冠- 6 和苯丙氨酸均为 $1 \times 10^{-4} \mathrm{~mol} / \mathrm{L}$, $\mathrm{Cu}^{2+}$ 浓度为 $2.5 \times 10^{-5} \mathrm{~mol} / \mathrm{L}$. 此溶液同时用于中心离 子为质子和中心离子为 $\mathrm{H}^{+}$和 $\mathrm{Cu}^{2+}$ 的手性识别实验.

\section{3 仪器条件}

毛细管电压为 $2.31 \mathrm{kV}$, 雉孔电压为 $35 \mathrm{~V}$, 管透 镜补偿电压为 $30 \mathrm{~V}$, 碰撞能量 $5 \mathrm{~V}$, 离子能量 $1 \mathrm{~V}$, 毛 细管温度 $100^{\circ} \mathrm{C}$, 脱溶剂温度 $180^{\circ} \mathrm{C}$; 鞘气为 $\mathrm{N}_{2}$, 流速 100 单位, $\mathrm{Ar}$ 为碰撞气体; 使用流动注射洜进样, 样 品流速为 $5 \mu \mathrm{L} / \mathrm{min}$. 所得质谱图均为 200 次累加得到 的平均值, 重复 3 次. 中心离子为 $\mathrm{H}^{+}$和 $\mathrm{Cu}^{2+}$ 的质谱 条件完全相同，具体条件见上.

\section{2 结果与讨论}

\section{1 当中心离子为 $\mathrm{H}^{+}$时的手性识别情况}

18-C-6-TCA 的结构式如图 1 所示. 18-C-6-TCA 为 18-C-6 的衍生物, 由于周围四个羧基的存在, 使之 具有四个手性中心从而产生了手性. 冠醚易于通过 氢键等非共价作用与对映体分子尤其是伯胺类化合物 形成非共价复合物. 而手性冠醚 18-C-6-TCA 由于具 有特定的构型, 使得它与对映体分子形成的非对映异 构复合物之间存在稳定性差异, 从而可用于手性识别.<smiles>O=C(O)C1OCCOCCOC(C(=O)O)C(C(=O)O)OCCOCCOC1C(=O)O</smiles>

\section{图 1 18-C-6-TCA 的结构式}

图 2(a) 和 (b) 分别为 (18-C-6-TCA) $(\mathrm{Phe}) \mathrm{H}^{+}$的 CID质谱图. 图中, $m / z 606$ 为复合物母离子, $m / z 441$ 为质子化的 18-C-6-TCA产物离子, 除此之外, 谱图 中还出现了较明显的 $m / z 395$ 次级产物离子. 比较图 2(a) 和 (b), 无论是母离子的裂解途径还是母离子的 解离效率, 两者都基本相同. 说明质子为中心离子时, 18-C-6-TCA对Phe对映体没有区分.

\section{2 当中心离子为 $\mathrm{Cu}^{2+}$ 时的手性识别情况}

图 3 (a) 和 (b) 是 18-C-6-TCA、Phe对映体和 $\mathrm{Cu}^{2+}$ 混合溶液的全扫描电喷雾质谱图. 图中, $m / z$ 

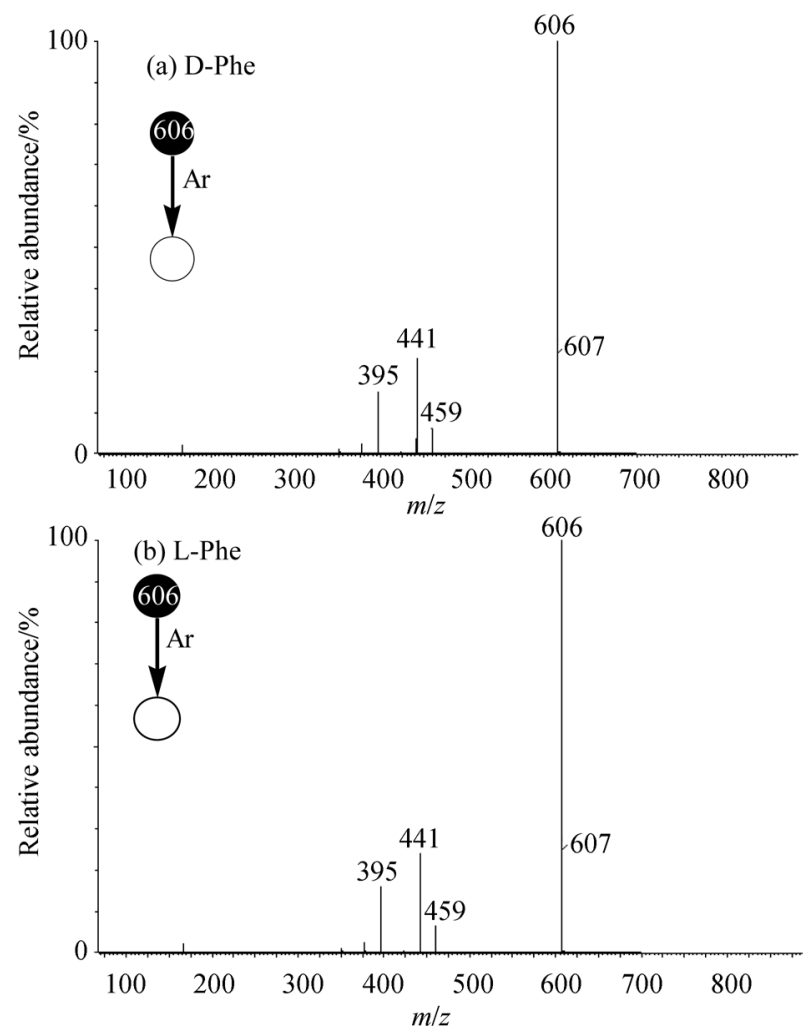

图 2 含(a) D-、(b) L-Phe的(18-C-6- TCA)(Phe) $\mathrm{H}^{+}$的CID 质谱图

18-C-6-TCA和Phe为 1: 1 混合, 浓度均为 $1 \times 10^{-4} \mathrm{~mol} / \mathrm{L}$, 碰撞能 量 $5 \mathrm{~V}$

606 为 (18-C-6-TCA)(Phe) $\mathrm{H}^{+}$(基峰), $m / z \quad 667$ 为 $\left[\mathrm{Cu}^{2+}(18-\mathrm{C}-6-\mathrm{TCA})(\mathrm{Phe})-\mathrm{H}^{+}\right]^{+}(<2 \%)$. 图 3 (a) 和 (b) 谱图完全一样，无法区分.

图 4 (a) 和(b) 分别是含 $D$ - 和 $L-P h e$ 的 $\left[\mathrm{Cu}^{2+}\right.$ (18-C-6-TCA)(Phe)- $\left.\mathrm{H}^{+}\right]^{+}$CID质谱图. 与图 2 (a) 和 (b) 明显不同的是, $\left[\mathrm{Cu}^{2+}(18-\mathrm{C}-6-\mathrm{TCA})(\mathrm{Phe})-\mathrm{H}^{+}\right]^{+}$只产 生 $\left[\mathrm{Cu}^{2+}(18-\mathrm{C}-6-\mathrm{TCA})-\mathrm{H}^{+}\right]^{+}(\mathrm{m} / \mathrm{z}$ 502), 而且是基峰. 这说明: (1) $\left[\mathrm{Cu}^{2+}(18-\mathrm{C}-6-\mathrm{TCA})(\mathrm{Phe})-\mathrm{H}^{+}\right]^{+}$的稳定性 较 (18-C-6-TCA) $(\mathrm{Phe}) \mathrm{H}^{+}$差, 因为前者CID谱图中产 物离子与母离子的相对丰度比明显高于后者；(2) 与 文献报道不同 ${ }^{[14]},\left[\mathrm{Cu}^{2+}(18-\mathrm{C}-6-\mathrm{TCA})(\mathrm{Phe})-\mathrm{H}^{+}\right]^{+}$的 $\mathrm{CID}$ 反应只有一个失去一分子 Phe的裂解反应。根据 相同反应条件下母离子的解离效率来区分 $D$-和 $L$-Phe.

对于形成加铜离子复合物的情况下, $R_{\text {chiral }}$ 可用 于衡量手性冠醚对于 $D$ 及 $L$-Phe的手性识别能力, $R_{\text {chiral }}$ 越偏离于 1 , 手性识别能力越强. $R_{\text {chiral }}$ 由下式 来表示:
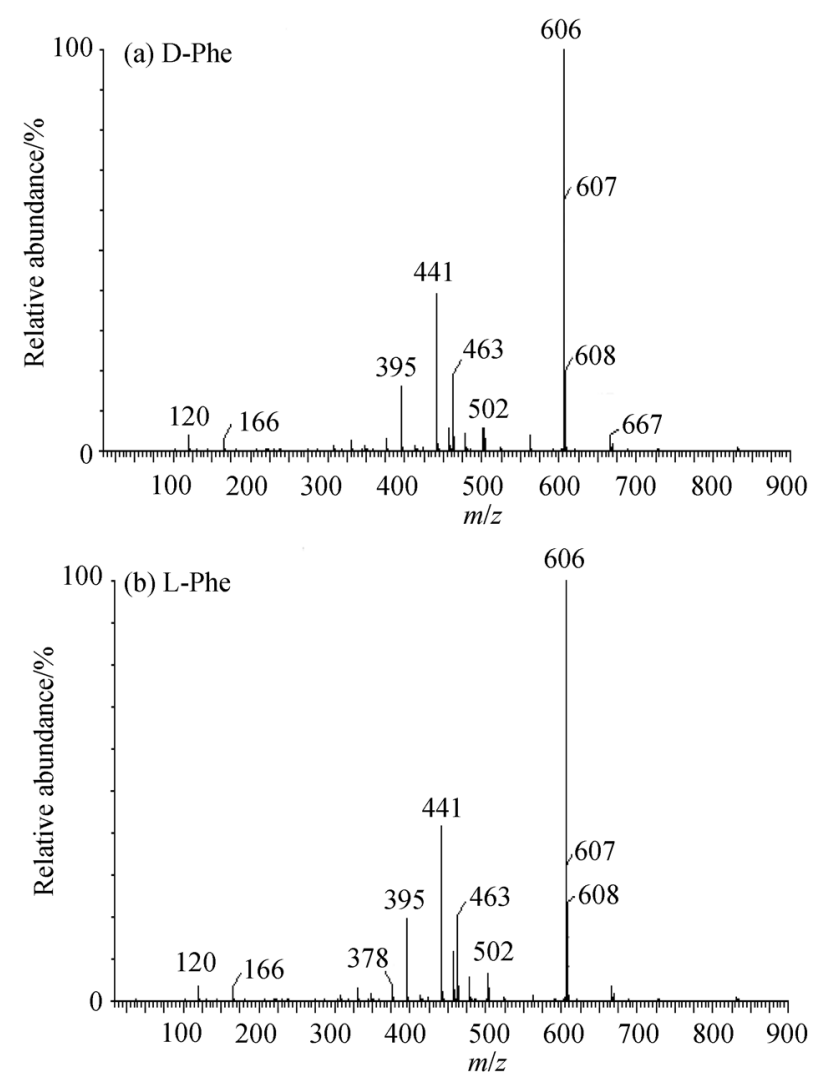

图 3 (a) D-和 (b) L-Phe、18-C-6-TCA和 $\mathrm{Cu}^{2+}$ 混合溶液 的全扫描电喷雾质谱图

18-C-6-TCA和Phe为 $1: 1$ 混合, 浓度均为 $1 \times 10^{-4} \mathrm{~mol} / \mathrm{L}, \mathrm{CuSO}_{4}$ 浓 度为 $2.5 \times 10^{-5} \mathrm{~mol} / \mathrm{L}$

$R_{\text {chiral }}=$

$\frac{\left[\mathrm{Cu}^{2+}(18-\mathrm{C}-6-\mathrm{TCA})-\mathrm{H}\right]^{+} /\left[\mathrm{Cu}^{2+}(\mathrm{Phe})_{\mathrm{D}}(18-\mathrm{C}-6-\mathrm{TCA})-\mathrm{H}\right]^{+}}{\left[\mathrm{Cu}^{2+}(18-\mathrm{C}-6-\mathrm{TCA})-\mathrm{H}\right]^{+} /\left[\mathrm{Cu}^{2+}(\mathrm{Phe})_{\mathrm{L}}(18-\mathrm{C}-6-\mathrm{TCA})-\mathrm{H}\right]^{+}}$, 由上述公式计算得: $R_{\text {chiral }}=0.83$. 而 $\mathrm{H}^{+}$的情况, $R_{\text {chiral }}$ $\approx 1$. 可见, 中心离子为 $\mathrm{Cu}^{2+}$ 时, 18-C-6-TCA对 Phe对 映体具有较强的识别能力. 以上结果表明, 中心离子 的选择确实与手性识别能力密切相关, 而 $\mathrm{Cu}^{2+}$ 离子 由于与多齿配体具有较强的配合作用使其手性识别 能力较强.

\section{3 由二聚体复合物的结构分析手性识别机制}

本课题组曾通过量化计算得到了质子化 $L$-色氨 酸与 18-冠-6 二聚体的最稳定构象. 质子化色氨酸中 的三个 $\mathrm{N}-\mathrm{H}$ 键象一个三角雉支撑在 18-冠-6 的平面之 上并与 18 -冠-6 中的氧原子形成三个 $\mathrm{N}-\mathrm{H} \cdots \mathrm{O}$ 氢键, 氢 键平均键长为 $1.929 \AA$, 平均键能为 $54.06 \mathrm{kcal} \cdot \mathrm{mol}^{-1}$. 

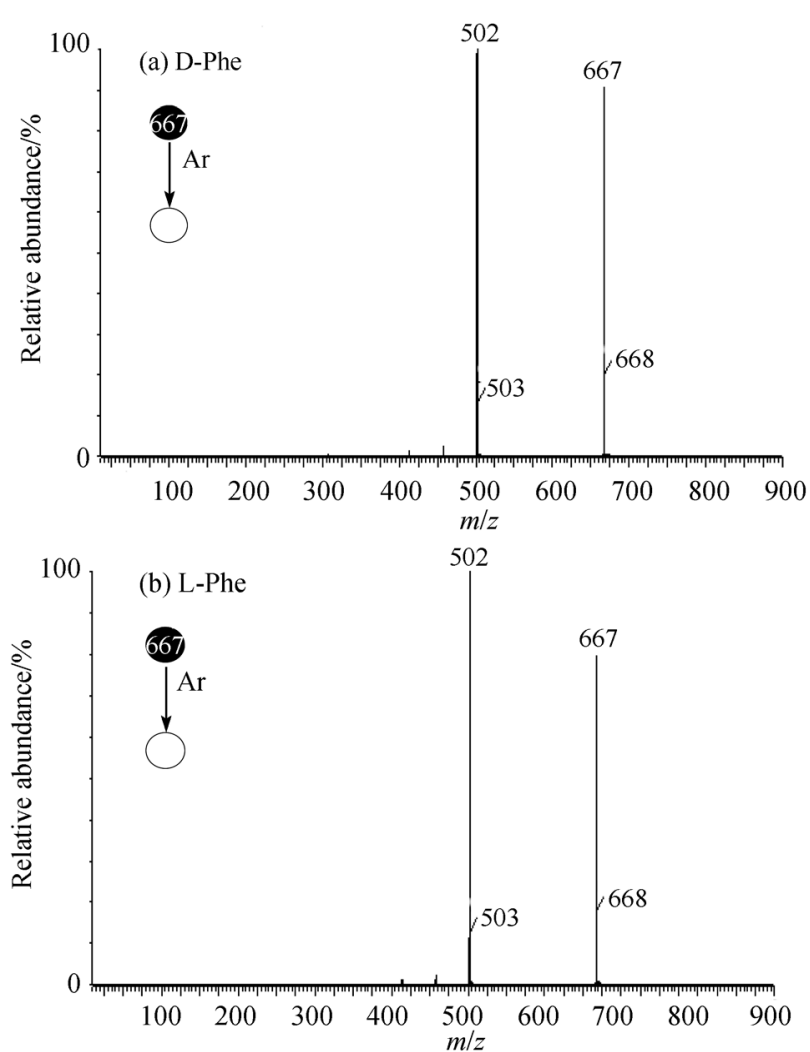

图 4 含 (a) $D$-、 (b) $L$-Phe $\left[\mathrm{Cu}^{2+}(18-C-6-\mathrm{TCA})(\mathrm{Phe})-\mathrm{H}^{+}\right]^{+}$ 的CID谱图

18-C-6-TCA和Phe为 $1: 1$ 混合, 浓度均为 $1 \times 10^{-4} \mathrm{~mol} / \mathrm{L}, \mathrm{CuSO}_{4}$ 浓 度为 $2.5 \times 10^{-5} \mathrm{~mol} / \mathrm{L}$, 碰撞能量 $5 \mathrm{~V}$

根据以上实验结果和文献[14], 我们提出了 $\mathrm{Cu}^{2+}$ 键合二聚体 $\left[\mathrm{Cu}^{2+}(18-\mathrm{C}-6-\mathrm{TCA})(\mathrm{Phe})-\mathrm{H}^{+}\right]^{+}$的构型(见 图 5), 该结构已通过量化计算证实: $\mathrm{Cu}^{2+}$ 离子位于 或接近于 18-C-6-TCA平面并分别与冠醚环中的两个 $\mathrm{O}$ 原子以及位于平面外的苯丙氨酸中的羧基中羟基 $\mathrm{O}$ 和氨基 $\mathrm{N}$ 原子形成四配位的离子型复合物. 这四个配 位键的键能差异较大，其中 $\mathrm{Cu}^{2+}$ 与 18-C-6-TCA冠醚 环中 $\mathrm{O}$ 的配位键能较强.

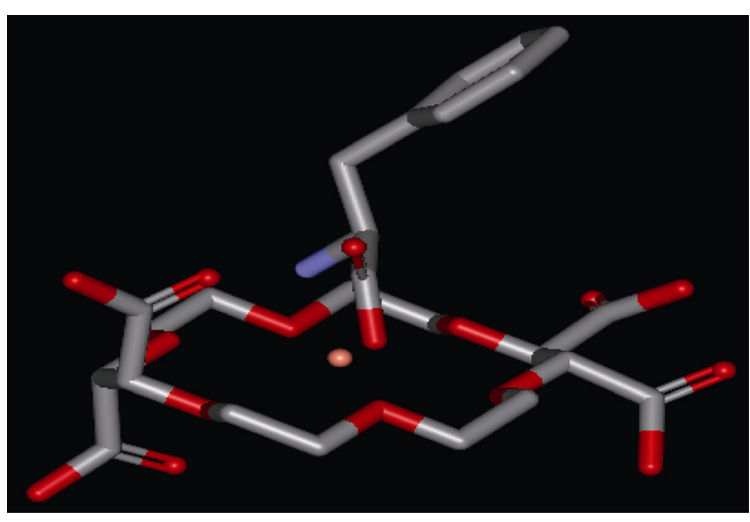

图 5 气相 $\left[\mathrm{Cu}^{2+}(18-\mathrm{C}-6-\mathrm{TCA})(\mathrm{Phe})-\mathrm{H}^{+}\right]^{+}$的结构示意图

通过 $\left[\mathrm{Cu}^{2+}\left(18-\mathrm{C}-6-\mathrm{TCA}(\mathrm{Phe})-\mathrm{H}^{+}\right)\right]^{+}$和 (18-6$\mathrm{TCA})(\mathrm{Phe}) \mathrm{H}^{+}$结构的描述，可以得出如下结论：(1) 质子键合二聚体的主要作用力是氢键. 由于氨基酸中 的 $\alpha-\mathrm{C}$ 上的烷基 $\mathrm{R}$ 垂直于 $18-\mathrm{C}-6-\mathrm{TCA}$ 平面并位于平面 的远端，两者之间的其他作用可忽略不计; (2) $\mathrm{Cu}^{2+}$ 键 合二聚体 $\left[\mathrm{Cu}^{2+}(18-\mathrm{C}-6-\mathrm{TCA})(\mathrm{Phe})-\mathrm{H}^{+}\right]^{+}$具有更多的 立体选择性. 主要作用力是 $\mathrm{Cu}^{2+}$ 分别与苯丙氨酸中苯 环和 18-C-6-TCA冠醚中杂原子的多齿型配位键. 除此 以外, 苯丙氨酸中苯环和 18-C-6-TCA中的羧基之间还 存在其他类型的非共价作用，如 $\pi-\pi$ 堆积作用等. 这种 作用虽然相对较弱, 但具有立体选择性, 是手性识别 的关键. 由于 $\left[\mathrm{Cu}^{2+}(18-\mathrm{C}-6-\mathrm{TCA})(\mathrm{Phe})-\mathrm{H}^{+}\right]^{+}$离子中 苯丙氨酸的苯环侧链与 18-C-6-TCA的其中一个羧基 在空间上比较靠近，这样的结构有利于苯环和羧基 之间的相互作用. $L$-和 $D$-苯丙氨酸对映体就是在这种 具有立体选择的弱作用力上表现出差异, 从而影响 整个离子的稳定性，成为手性识别的前提. 总之， $\mathrm{Cu}^{2+}$ 键合二聚体复合物 $\left[\mathrm{Cu}^{2+}(18-\mathrm{C}-6-\mathrm{TCA})\right.$ $\left.(\mathrm{Phe}) \mathrm{H}^{+}\right]^{+}$是从络合空间上对苯丙氨酸对映体进行立 体识别, 其手性识别能力要较 $\mathrm{H}^{+}$强得多.

致谢本工作得到中国科学院院长基金和教育部科学技术研究重点项目(编号: 106064)资助.

\section{参考文献}

1 Lucey J F, Behrman R B. Thalidomide: Effect upon pregnancy in the rhesus Monkey. Science, 1963, 139: 1295-1296[DOI]

2 Kyba E P, Timko J M, Kaplan L J, Jong F G, Gokel G W, Cram D J. Host-guest complexation. 11. Survey of chiral recognition of amine and amino ester salts by dilocular bisdinaphthyl hosts. J Am Chem Soc, 1987, 100(14): 4555—4568 [DOI] 
3 Bour P, Kim J, Kapitan J, Hammer R P, Huang R, Wu L, Keiderling T A. Vibrational circular dichroism and IR spectral analysis as a test of theoretical conformational modeling for a cyclic hexapeptide. Chirality, 2008, 20(10): 1104-1119 [DOI]

4 Finn M G. Emerging methods for the rapid determination of enantiomeric excess. Chirality, 2002, 14(7): 534-540[DOI]

5 Schmidt R, Wahl H G, Häberle H, Dieterich H -J, Schurig V. Headspace gas chromatography-mass spectrometry analysis of isoflurane enantiomers in blood samples after anesthesia with the racemic mixture. Chirality, 1999, 11(3): 206-211 [DOI]

6 Koppenhoefer B, Zhu X F, Jakob A, Wuerthner S, Lin B C. Separation of drug enantiomers by capillary electrophoresis in the presence of neutral cyclodextrins. J Chromatogr A, 2000, 875: 135-161[DOI]

7 McLafferty F. Tandem mass spectrometry. Science, 1981, 214: 280-287 [DOI]

8 Sawada M, Takai Y, Yamada H, Nishida J, Kaneda T, Arakawa R, Okamoto M, Hirose K, Tanakab T, Naemura K. Chiral amino acid recognition detected by electrospray ionization (ESI) and fast atom bombardment (FAB) mass spectrometry (MS) coupled with the enantiomer-labelled (EL) guest method. J Chem Soc, Perkin Trans 2, 1998, 2(3): 701-710 [DOI]

9 Sawada M, Okumura Y, Yamada H, Takai Y, Takahashi S, Kaneda T, Hirose K, Misumi S. Cross-chiral examinations of molecular enantioselective recognition by fast atom bombardment mass spectrometry: Host-guest complexations between chiral crown ethers and chiral organic ammonium ions. Org Mass Spectrom, 1993, 28(12): 1525-1528 [DOD]

10 Sawada M, Takai Y, Yamada H, Hirayama S, Kaneda T, Tanaka T, Kamada K, Mizooku T, Takeuchi S, Hirose K, Tobe Y, Naemura K. Chiral recognition in host-guest complexation determined by the enantiomer-labeled guest method using fast atom bombardment mass spectrometry. J Am Chem Soc, 1995, 117(29): 7726-7736[DOI]

11 Fales H M, Wright G J. Detection of chirality with the chemical ionization mass spectrometer. “Meso” ions in the gas phase. J Am Soc Mass Spectrom, 1977, 99(7): 2339-2340

12 Zhang D X, Tao W A, Cooks R G. Chiral resolution of $D$-and $L$-amino acids by tandem mass spectrometry of Ni( II )-bound trimeric complexes. Int J Mass Spectrom, 2001, 204(1-3): 159-169 $\underline{\text { DOI }]}$

13 Wu L M, Tao A W, Cooks R G. Ligand and metal-ion effects in metal-ion clusters used for chiral analysis of $\alpha$-hydroxy acids by the kinetic method. Anal Bioanal Chem, 2002, 373: 618-627 [DOI]

14 Tao W A, Zhang D, Nikolaev E N, Cooks R G. Copper(II)-assisted enantiomeric analysis of $D, L$-amino acids using the kinetic method: Chiral recognition and quantification in the gas phase. J Am Chem Soc, 2000, 122(43): 10598 - 10609 [DOI]

15 Yao ZP, Wan T S M, Kwong K P, Che C T. Chiral analysis by electrospray ionization mass spectrometry/mass spectrometry. 1. Chiral recognition of 19 common amino acids. Anal Bioanal Chem, 2000, 72(21): 5383-5393

16 Tao W A, Zhang D, Wang F, Thomas P D, Cooks R G. Kinetic resolution of $D, L$-amino acids based on gas-phase dissociation of copper( II ) complexes. Anal Chem, 1999, 71(19): 4427-4429 [DOD]

17 Tao A W, Gozzo F C, Cooks R G. Mass spectrometric quantitation of chiral drugs by the Kinetic method. Anal Chem, 2001, 73(8): $1692-1698[\mathrm{DOI}]$

18 Tao W A, Cooks R G. Peer Reviewed: Chiral analysis by MS. Anal Chem, 2003, 75(1): 25 A-31 A[DOI]

19 Cheng Y, Hercules D M. Measurement of chiral complexes of cyclodextrins and amino acids by electrospray ionization time-of-flight mass spectrometry. J Mass Spectrom, 2001, 36(7): 834-836 $\underline{\text { [DOI] }}$

20 Grigorean G, Ramirez J, Ahn S H, Lebrilla C B. A mass spectrometry method for the determination of enantiomeric excess in mixtures of D,L-amino acids. Anal Chem, 2000, 72(18): 4275-4281 [DOI]

21 Grigorean G, Gronert S, Lebrilla C B. Enantioselective gas-phase ion-molecule reactions in a quadrupole ion trap. Int J Mass Spectrom, 2002, 219(1): 79-87[DOH]

22 Grigorean G, Cong X, Lebrilla C B. Chiral analyses of peptides by ion/molecule reactions. Int J Mass Spectrom, 2004, 234(1-3): $71-77 \underline{[\mathrm{DOI}]}$

23 Ramirez J, He F, Lebrilla C B. Gas-phase chiral differentiation of amino acid guests in cyclodextrin hosts. J Am Chem Soc, 1998, 120(29): 7387-7388 $\underline{\text { [DOI] }}$

24 François Gal J, Stone M, Lebrilla C B. Chiral recognition of non-natural $\alpha$-amino acids. Int J Mass Spectrom, 2003, 222(1-3): $259-267 \underline{[\mathrm{DOI}]}$

25 Grigorean G, Lebrilla C B. Enantiomeric analysis of pharmaceutical compounds by ion/molecule reactions. J Am Chem Soc, 2001, 73(8): 1684-1691

26 Yu C T, Guo Y L, Chen G Q, Zhong Y W. Chiral recognition of zinc(II) ion complexes composed of bicyclo[3.3.0] octane-2,6-diol and s-naproxen probed by collisional-induced dissociation. J Am Soc Mass Spectrom, 2004, 15(6): 795-802 [DOI] 
27 Lu H J, Guo Y L. Chiral recognition of borneol by association with zinc( II) and -tryptophan in the gas phase. Anal Chim Acta, 2003, 482(1): $1-7[\mathrm{DOI}]$

28 李明，陈焕文，刘志强，刘淑莹，金钦汉. 质谱动力学方法在手性分析中的应用. 化学进展, 2006, 18(10): 1369-1374

29 刘勤，张淑珍，谢剑炜，吴弼东，申㤐，刘克良。电喷雾质谱在手性识别和分析中的应用. 化学进展, 2006, 18: 780-788

30 Liu Q, Zhang S Z, Wu B D, Guo J F, Xie J W, Gu M S, Zhao Y M, Yun L H, Liu K L. Chiral melamine derivatives: design, synthesis, and application to mass spectrometry-based chiral analysis. Anal Chem, 2005, 77(16): 5302 - 5310[DOI]

$31 \mathrm{Lu} \mathrm{H} \mathrm{J,} \mathrm{Guo} \mathrm{Y} \mathrm{L.} \mathrm{Evaluation} \mathrm{of} \mathrm{chiral} \mathrm{recognition} \mathrm{characteristics} \mathrm{of} \mathrm{metal} \mathrm{and} \mathrm{proton} \mathrm{complexes} \mathrm{of} \mathrm{di-o-benzoyl-tartaric} \mathrm{acid} \mathrm{dibutyl}$ ester and L-tryptophan in the gas phase. J Am Soc Mass Spectrom, 2003, 14(6): 571-580[DOI]

32 Wu L M, Tao W A, Cooks R G. Kinetic method for the simultaneous chiral analysis of different amino acids in mixtures. J Mass Spectrom, 2003, 38(4): 386-393[DOI]

33 Arakawa R, Kobayashi M, Fukuo T, Shiraiwa T. Rapid Commun. Studies on the association of 2-thiazolidinecarboxylic acid and antimony potassium tartrate: chiral recognition and prediction of absolute configuration by electrospray ionization mass spectrometry, Rapid Commun Mass Spectrom, 2001, 15(9): 685-689 [DOI]

34 Arakawa R, Kobayashi M, Ama T. Chiral recognition in association between antimony potassium tartrate and bis(L-alaninate)ethylenediamine cobalt(III) complexes using electrospray ionization mass spectrometry. J Am Soc Mass Spectrom, 2000, 11(9): $804-808 \underline{[\mathrm{DOI}]}$

35 宋凤瑞，刘子阳，孙维星，刘淑莹、区分麻黄碱立体异构体的质谱新方法. 分析化学, 1999, 27(9): 1000-1002

36 于湛, 间存玉，宋风瑞，刘志强，刘淑莹。七-(2，6-二-O-甲基)- $\beta$-环糊精对薄荷醇对映体手性识别的电喷雾质谱研究，分析 化学, 2006, 34(5): 671-674

37 于湛, 问存玉, 宋风瑞, 刘志强, 刘淑莹. (2, 6-二-O-甲基)- $\beta$-环糊精对 $1,1^{\prime}$-联菜酚对映体手性识别的电喷雾质谱研究. 化学 学报, 2006, 64(14): 1507-1512

38 Schug K, Lindner W. Stereoselective discrimination and quantification of arginine and N-blocked arginine enantiomers by formation and dissociation of calcium-mediated diastereomeric trimer complexes with a chiral reference compound using electrospray ionization-ion trap tandem mass spectrometry. J Am Soc Mass Spectrom, 2005, 16(6): 825-834[DOI] 